\title{
Vers une procédure de contrôle des structures en verre trempé
}

\author{
Fabrice Bernard ${ }^{1}$, Laurent Daudeville ${ }^{2, a}$ et René Gy ${ }^{3}$ \\ 1 École des mines de Douai, Département Génie Civil, 941 rue Charles Bourseul, 59508 Douai Cedex, France \\ 2 Laboratoire Sols Solides Structures, Domaine Universitaire, 38041 Grenoble Cedex 9, France \\ 3 Saint-Gobain Recherche, 39 quai Lucien Lefranc, 93303 Aubervilliers Cedex, France
}

Reçu le 4 décembre 2002, accepté le 5 décembre 2003

\begin{abstract}
Résumé - Ce travail porte sur l'utilisation du verre comme véritable matériau de structure et concerne plus particulièrement la proposition d'une méthode de contrôle in situ non destructif des structures en verre trempé thermiquement, notamment dans les zones d'assemblage. Une méthode de dimensionnement de la structure est proposée. Elle est fondée d'une part, sur un état limite ultime garantissant la pérennité de la structure sur une longue durée de chargement, et d'autre part, sur une prédiction, par la méthode des éléments finis, en tout point de la structure, de l'état de contraintes résiduelles dues au procédé de trempe thermique ainsi que celles dues au chargement mécanique extérieur. La méthode de contrôle consiste à comparer, durant la vie de la structure, des images issues d'analyses photoélastiques sur la structure chargée, à des images obtenues par calcul aux éléments finis pour l'état limite ultime ayant servi au dimensionnement.
\end{abstract}

Mots clés : Verre trempé / structures / mesures / contrôle in situ / photoélasticité / éléments finis / contraintes résiduelles / état limite ultime

\begin{abstract}
Towards a control process for tempered glass structures. This work deals with the use of glass as a real structural material, and more particularly concerns a proposal of an in situ non destructive control process for tempered glass structures, especially in joint zones. A design method is proposed. It is based on one hand, on a limit state ensuring the structure perenniallity on long duration, and on another hand, on the finite element prediction, on every point of the structure, of both residual stresses due to tempering and stresses due to the mechanical loading. The control method consists, during the life of the structure, in comparing images obtained from photoelastic analyses of the loaded structure, to images obtained from finite element analyses of the structure at the ultimate limit state used for the design.
\end{abstract}

Key words: Tempered glass / structures / measurements / in situ control / photoelasticity / finite elements / residual stresses / ultimate limit state

\section{Introduction}

L'emploi du verre pour les structures du Génie Civil est fortement pénalisé. En effet, en dehors de qualités indéniables, comme une excellente durabilité ou une résistance mécanique intrinsèque très élevée, le verre présente des inconvénients certains : c'est d'abord un matériau fragile, très sensible à l'endommagement mécanique de sa surface. La moindre fissuration superficielle, quelle qu'en soit l'origine, fera chuter la résistance de plusieurs ordres de grandeur. C'est ensuite un matériau présentant un phénomène de propagation sous-critique de fissure [1] ; pour une valeur du facteur d'intensité des

\footnotetext{
a Auteur correspondant : laurent.daudeville@inpg.fr
}

contraintes inférieure à la valeur critique (la ténacité) et pour un chargement constant, une lente propagation des défauts de surface a lieu (en raison d'une réaction chimique en pointe de fissure entre le verre et l'humidité ambiante).

C'est pour ces raisons que les bureaux de contrôle pour la construction exigent des cœefficients partiels de sécurité relativement importants et des essais en vraie grandeur pour toute structure en verre à réaliser. Les coefficients partiels de sécurité proviennent :

- de cofficients intégrant les incertitudes sur les charges, - de coefficients intégrant les incertitudes sur le comportement du matériau à court et long terme. 


\section{Nomenclature}

\begin{tabular}{|ll|}
\hline $\mathrm{A}$ & vecteur analyseur \\
$\mathrm{C}$ & constante photoélastique du verre \\
$\mathrm{E}$ & vecteur champ électrique \\
$\mathrm{E}_{0}$ & vecteur champ électrique émis \\
$\mathrm{E}_{1}, \mathrm{E}_{2}$ & coordonnées du vecteur champ électrique \\
$\mathrm{h}$ & épaisseur de matériau traversé par le rayon lumineux \\
$\mathrm{I}$ & intensité de la lumière \\
$\mathrm{I}_{0}$ & intensité de lumière émise \\
$\mathrm{I}_{\mathrm{d}}$ & matrice identité \\
$\mathrm{M}$ & opérateur d'intégration du champ électrique \\
$\mathrm{n}_{1}, \mathrm{n}_{2}$ & indices optiques \\
$\mathrm{T}$ & température du verre \\
$x_{3}$ & coordonnée selon l'épaisseur de la plaque de verre \\
$\Delta$ & retard de phase \\
$\varphi$ & angle entre la première direction principale secondaire et l'axe du polariseur \\
$\kappa$ & angle intermédiaire \\
$\lambda$ & longueur d'onde de la vibration lumineuse \\
$\sigma_{1}, \sigma_{2}$ & contraintes principales secondaires \\
$\sigma_{11}, \sigma_{12}, \sigma_{22}$ & composantes dans le plan du tenseur des contraintes \\
$\xi$ & temps réduit \\
\hline
\end{tabular}

Un cœfficient partiel de sécurité de 2 est couramment utilisé pour la prise en compte du comportement à long terme du verre, il provient de l'estimation de la perte de résistance du verre sur des durées de vie de bâtiments (50 ans). La diminution de la résistance pour de grandes durées de chargement est due au phénomène de fissuration sous-critique. Ce cofficient partiel de sécurité est très pénalisant, il peut contribuer à dissuader l'utilisation du verre comme matériau de structure.

Pour diminuer la sensibilité du verre aux endommagements de surface, il existe des méthodes de renforcement, la plus intéressante en vue d'applications structurelles étant la trempe thermique qui consiste à refroidir brutalement, par jets d'air, un verre chauffé à plus de $600{ }^{\circ} \mathrm{C}$. On obtient alors une mise en compression de la surface du matériau (de 100 à $300 \mathrm{MPa}$ couramment); la résistance est donc augmentée puisque pour casser le verre il faudra d'abord vaincre cette pré-compression de surface, et on obtient une certaine immunité vis-à-vis de la fissuration sous-critique tant que les défauts de surface ne sont pas ouverts, c'est-à-dire tant que les contraintes appliquées n'excèdent pas en valeur absolue la pré-compression surfacique. Néanmoins les bureaux de contrôle exigent des essais en vraie grandeur pour la validation expérimentale du dimensionnement des structures en verre trempé en raison du manque d'information sur l'état de contraintes résiduelles généré par le processus de trempe.

Des études antérieures ont montré que l'on avait répondu aux principales questions concernant le vieillissement des structures en verre trempé puisque la pérennité des précontraintes de trempe devrait être garantie sur 50 années [2].

Compte tenu de ces remarques, il apparaît possible de favoriser l'utilisation du verre trempé dans les structures. En effet, si d'une part, il est garanti que la surface du verre n'est jamais en traction, il est alors possible de s'affranchir du coefficient partiel de sécurité égal à 2. Si d'autre part, les concepteurs sont en mesure de calculer l'état de contraintes en tout point de la structure soumise à un chargement typique de ceux rencontrés en Génie Civil, et de dimensionner la structure en verre à l'état limite de décompression de la surface, alors les essais en vraie grandeur ne sont plus nécessaires. Si, en outre, une procédure de contrôle de ces structures est proposée, permettant de vérifier in situ que les charges de décompression de surface ne sont pas atteintes, alors il sera possible de dresser des certificats de garantie. Un assouplissement des méthodes actuelles de dimensionnement des structures en verre pourrait ainsi voir le jour.

Cet article présente une méthodologie de contrôle in situ des structures en verre trempé. Dans un premier temps, les outils de calcul des contraintes seront rapidement présentés, puis un moyen de contrôle non destructif des structures en verre, reposant sur une utilisation originale de la photoélasticité, sera développé plus longuement permettant de vérifier si un état de contraintes donné est atteint.

\section{Les outils de calcul des états de contraintes dans les structures en verre}

Les figures 1 et 2 montrent un exemple de structure innovante en verre trempé. Il s'agit d'une voûte en verre trempé, au-dessus d'un accès à une station de transport souterrain, réalisée en 1996 près du forum international de Tokyo au Japon.

C'est la plus grande structure en verre au monde, ne présentant aucun support métallique. Chaque poutre est constituée de deux lames en verre trempé. Le feuilletage est assuré par un joint en Polyvinyle de Butyral (PVB). La plus grande poutre fait cinq mètres. Les assemblages sont réalisés par des connecteurs métalliques traversant un trou de la plaque de verre. Chaque poutre comporte 


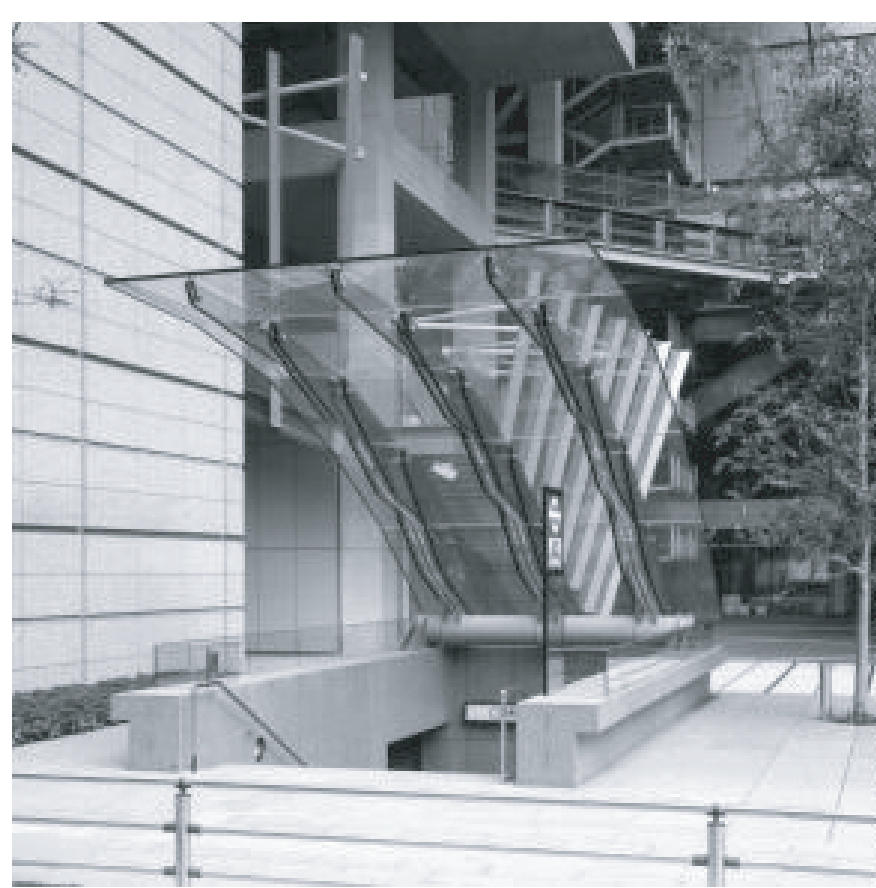

Fig. 1. Voûte en verre trempé du forum international de Tokyo.

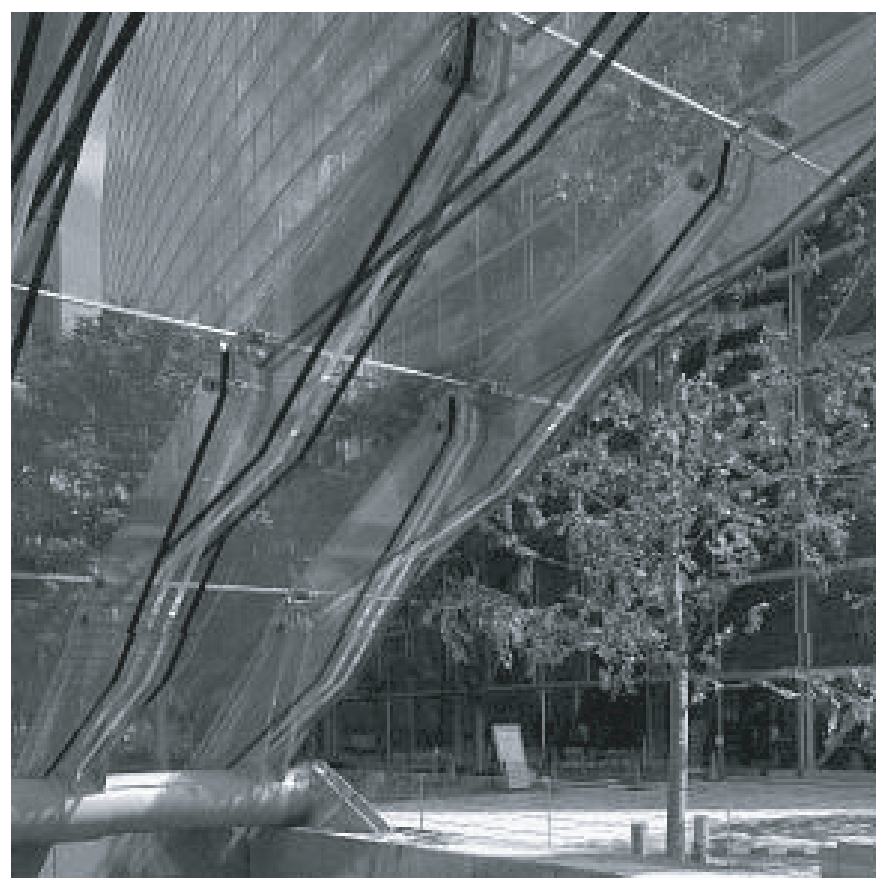

Fig. 2. Détail des poutres et assemblages de la voûte en verre trempé.

quatre trous. Ce type de technologie est celle actuellement utilisée pour les Vitrages Extérieurs Accrochés (VEA) (Fig. 3 d'après [3]). Le trou dans le verre peut être chanfreiné ou non. Un matériau suffisamment ductile permettant d'éviter une concentration de contrainte sur la surface chanfreinée est intercalé entre le verre et l'acier. Il est en général en aluminium car ce matériau a des propriétés

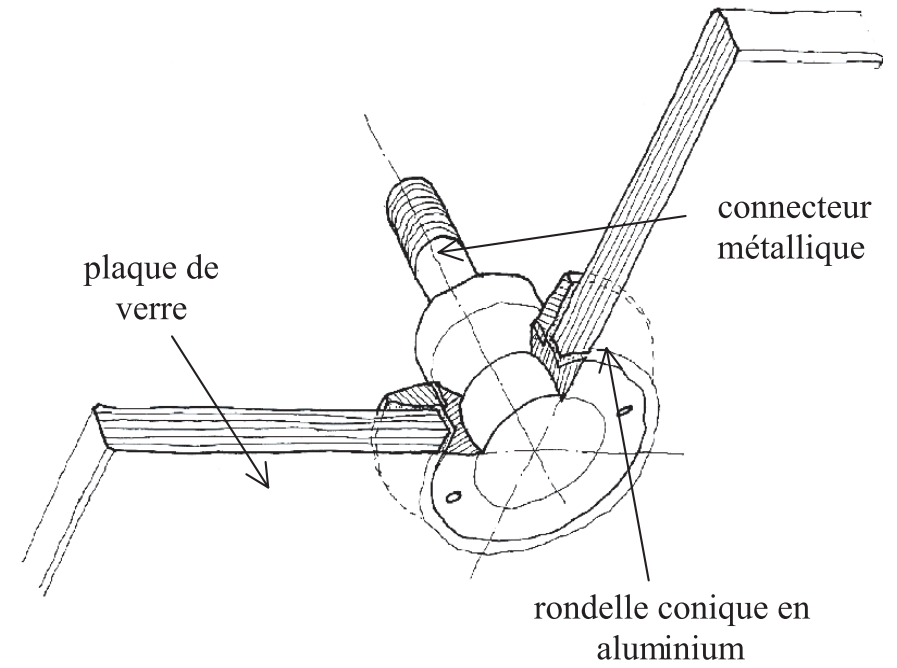

Fig. 3. Schéma de l'assemblage (de type VEA).

élastiques quasiment identiques à celles du verre et peut se déformer de façon plastique pour des contraintes assez faibles. La réalisation de cette structure a nécessité une campagne expérimentale de grande ampleur.

Au travers de cet exemple, il peut être constaté que les éléments en verre plat utilisés sont chargés dans leur plan de manière prolongée, la direction perpendiculaire offrant peu de rigidité. L'action mécanique appliquée par l'assemblage sur la plaque de verre trempée est une force. C'est le type de chargement rencontré dans les applications structurales utilisant du verre trempé.

La prédiction de la capacité portante d'une structure en verre trempé nécessite de distinguer les zones courantes des zones d'assemblage, pour lesquelles les états de contraintes, qu'ils soient dus au chargement extérieur ou bien résiduels de trempe, sont complètement différents.

L'état de contraintes en tout point d'une structure en verre trempé est la superposition de ces deux champs de contraintes. Pour les appréhender, il est proposé d'avoir recours à la modélisation par Éléments Finis.

\subsection{Calcul des contraintes résiduelles}

Le calcul à réaliser est un calcul thermomécanique découplé; il est par conséquent nécessaire de connaître le comportement mécanique complexe du verre au cours de la trempe (un phénomène faisant intervenir à la fois le temps et la température) ainsi que l'historique des températures pendant le processus.

Aux températures ambiantes jusqu'à $450{ }^{\circ} \mathrm{C}$ environ, le verre se comporte comme un solide élastique linéaire isotrope. Aux températures élevées (supérieures à $650^{\circ} \mathrm{C}$ ), il a les propriétés d'un fluide visqueux. Le passage d'un comportement à l'autre se fait de manière continue : on n'observe pas de fusion franche comme pour le cas des solides cristallins mais plutôt un phénomène de ramollissement progressif. Dans ce domaine intermédiaire, le verre montre un comportement viscoélastique, thermorhéologiquement simple et soumis à la relaxation 
structurale. Pour décrire ce comportement, le modèle de Narayanaswamy [4] fait office de référence. Il permet une implantation dans un code aux Éléments Finis [5]. Le comportement viscoélastique est modélisé par l'intermédiaire d'un modèle de Maxwell généralisé. La simplicité thermorhéologique traduit le fait que la température et le temps ne sont pas deux variables d'état indépendantes. Le verre présente le même comportement à différentes températures. Ainsi, si le comportement du verre est connu à une température de référence, il peut être déterminé à n'importe quelle autre température. L'élimination d'une variable entre le temps et la température se fait par l'intermédiaire du temps réduit $\xi(\mathrm{T})$ défini comme le rapport de la viscosité à la température $\mathrm{T}$ et la viscosité à la température de référence. La relaxation structurale est une conséquence directe de la définition thermodynamique d'un verre, et traduit le fait que, suivant la vitesse de refroidissement, le verre n'aura pas le même état structural. Ce phénomène est pris en compte grâce à la notion de température fictive, qui représente la température du liquide qui est dans le même état structural que le verre en question. Le modèle de Narayanswamy considère que température réelle et température de référence jouent le même rôle.

Les échanges thermiques intervenant pendant le processus de trempe (conduction et surtout convection forcée et rayonnement dans un milieu semi-transparent) sont soigneusement identifiés dans [6] que l'on soit loin des bords et du trou (problème 1D), près des bords (problème 2D) ou près du trou (problème $3 \mathrm{D})$. Le rayonnement thermique est pris en compte dans la simulation numérique par le biais d'un modèle à deux émissivités (surfacique et volumique) dont la pertinence a été démontrée pour des plaques de grande épaisseur et pour les zones proches des bords et des trous. Les coefficients de convection ont été identifiés de manière expérimentale en élaborant une maquette creuse en aluminium représentant les surfaces extérieures d'une plaque de verre trouée puis en lui faisant subir un refroidissement de trempe sur une ligne de production du groupe Saint-Gobain. Cela permet un calcul précis des contraintes résiduelles de trempe [7].

\subsection{Calcul des contraintes appliquées}

Dans les zones courantes des poutres précédentes, ou pour toute autre poutre plus « classique », le champ des contraintes appliquées se calcule aisément (poutres en flexion) sans avoir recours à des techniques numériques. En revanche, pour les zones de connexion, on observe une localisation de contraintes tridimensionnelles. Des expressions littérales des états de contraintes dans ces zones, pour des chargements plans, peuvent se trouver dans le cas de connecteurs et de trous cylindriques sans matériau intercalaire [8], hypothèses trop restrictives pour notre étude (présence de chanfrein, bague intermédiaire en aluminium. . .). Il est donc nécessaire d'avoir recours de nouveau à la simulation par Éléments Finis. La figure 4 présente une photo et une coupe du connecteur étudié. Le problème rencontré est un problème de contact entre
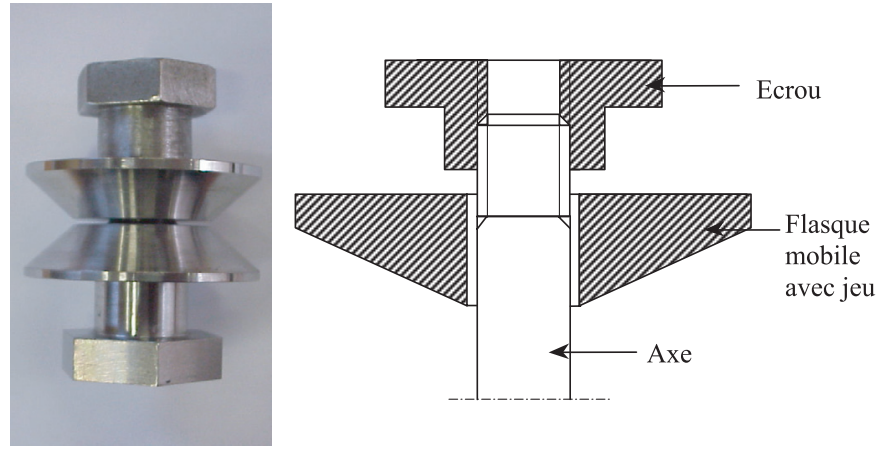

Fig. 4. Connecteur en acier.

des objets tridimensionnels en mouvement possible les uns par rapport aux autres. Ces mouvements sont des glissements avec frottement. Deux contacts différents se produisent : entre la bague en aluminium et le chanfrein du verre, et entre cette même bague et le connecteur métallique. Le problème est rendu compliqué par la présence de jeux entre les objets, mais aussi dans les objets (par exemple, le connecteur métallique est composé de différentes parties : un axe cylindrique et une bague conique (Fig. 4)). La résolution de ce problème a été menée avec le code aux éléments finis Abaqus, la gestion des contacts repose sur la méthode des projections [9]. Les résultats de simulations ainsi que les hypothèses retenues sont présentés dans [6]. La modélisation a été validée expérimentalement grâce à une grande campagne d'essais menée parallèlement : les états de contraintes au sein de plaques de verre trempé chargées jusqu'à rupture ont été retrouvés avec une bonne précision [6].

\subsection{Superposition}

En superposant les deux états de contraintes déterminés précédemment, il est ainsi possible de déterminer en tout point de la structure en verre trempé l'état de contraintes généré par le chargement du connecteur métallique sur le verre trempé thermiquement. Il est alors proposé de calculer pour quel chargement la décompression de la surface est atteinte. Dans [6] les charges de décompression de surface sont données en fonction du pré-serrage imposé du connecteur sur la bague en aluminium et le verre et en fonction de la géométrie de trou, pour un chargement s'effectuant dans le plan de la plaque. Ces charges sont alors les limites à considérer pour le dimensionnement.

\section{Un moyen de contrôle in situ : la photoélasticité}

La transparence du verre et son caractère biréfringent, lorsqu'il est sollicité, sont deux propriétés uniques dans le domaine des matériaux de construction qu'il est proposé d'utiliser de façon originale. 
Pour cela, il est nécessaire d'être en mesure de passer d'une image photoélastique à un état de contraintes, et réciproquement. Par la suite, les principes de la photoélasticité et de la polariscopie seront rappelés, puis sera présentée une méthode de simulation des images photoélastiques basée sur l'exploitation des résultats de simulations par Éléments Finis expliquées précédemment. La difficulté provient du caractère tridimensionnel des contraintes dans les zones d'assemblage qui rend non classique l'analyse photoélastique.

\subsection{Polariscopie et photoélasticité}

\subsubsection{Définition de la biréfringence}

La théorie électromagnétique due à Maxwell est souvent utilisée pour décrire la nature ondulatoire de la lumière. Cette théorie stipule que la lumière se propage comme des ondes électromagnétiques transversales. Le vecteur lumineux, perpendiculaire à la direction de propagation, s'identifie ainsi à deux grandeurs vectorielles, en phase et orthogonales entre elles : le champ électrique et le champ magnétique. On montre que l'étude du champ électrique E, seul, suffit pour décrire la lumière. L'intensité I de la lumière est égale au carré de la norme du vecteur champ électrique E [10].

Le rayon lumineux émis par la source n'est pas contraint à suivre une orientation privilégiée. Il est composé d'un nombre arbitraire de vibrations transversales. La lumière se propage toujours de façon à ce que le temps de parcours soit le plus grand ou le plus petit possible. Ainsi, qu'elle que soit la direction de la vibration au moment où elle pénètre dans un milieu transparent anisotrope, cette vibration se divise immédiatement en deux composantes orthogonales dirigées respectivement suivant les directions de plan d'onde pour lesquelles l'indice optique est le plus grand et le plus petit possible. Les matériaux qui souscrivent à cette règle sont dits biréfringents. Ils transforment la vibration lumineuse en deux composantes orthogonales qui ne se déplacent pas à la même vitesse dans le matériau, et qui émergent avec un retard relatif égal à :

$$
\Delta=\left(\frac{2 \pi}{\lambda}\right) \mathrm{h}\left(\mathrm{n}_{2}-\mathrm{n}_{1}\right)
$$

$\mathrm{n}_{2}$ et $\mathrm{n}_{1}$ sont les indices optiques des deux directions de vibration, $\mathrm{h}$ est l'épaisseur du matériau et $\lambda$ est la longueur d'onde de la vibration.

Lorsqu'une lame biréfringente est faite de telle sorte qu'elle donne un retard relatif de $\frac{\pi}{2}$, elle est appelée lame quart d'onde.

\subsubsection{Polariscopie et photoélasticité}

Un polariscope plan se compose d'une source de lumière, blanche ou monochromatique, et de deux polariseurs. À cela, on ajoute deux lames quart d'onde pour

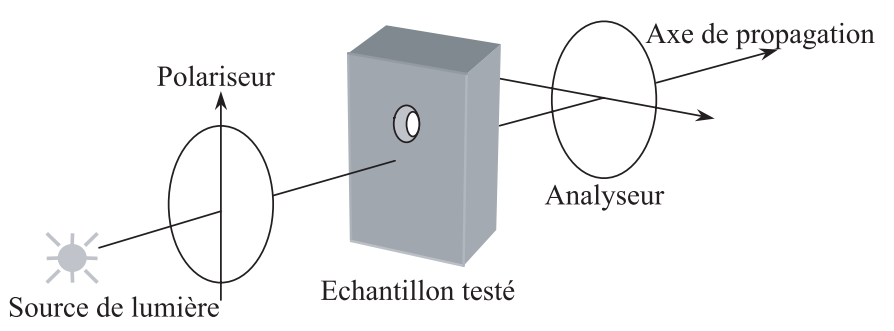

Fig. 5. Schéma de principe d'un polariscope plan.

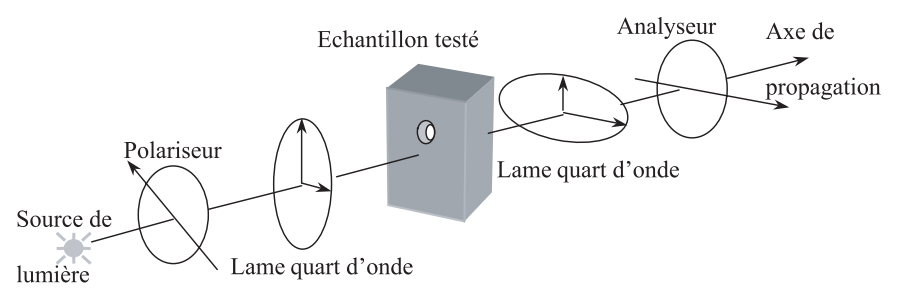

Fig. 6. Schéma de principe d'un polariscope circulaire.

faire un polariscope circulaire. L'élément de verre à analyser se place entre les deux polariseurs ou les deux lames quart d'onde (Figs. 5 et 6 ).

Le rayon lumineux, émis par la source, traverse dans un premier temps le premier polariseur. Il est alors astreint à vibrer dans un plan normal à la direction de propagation déterminé par l'orientation du polariseur. Le rayon lumineux est polarisé rectilignement. Si une lame quart d'onde est présente, l'amplitude du vecteur lumineux émergent est constante, et la pointe du vecteur dessine un cercle lorsque l'onde se propage : le rayon lumineux se propage circulairement. Le rayon lumineux continue alors de se propager et rencontre le verre. Celuici, comme plusieurs autres matériaux transparents non cristallins, est isotrope optiquement sous conditions normales mais devient biréfringent, comme un cristal, lorsqu'il est sollicité. Ce phénomène est appelé biréfringence accidentelle ou photoélasticité.

Les propriétés optiques du verre peuvent être représentées en chaque point par un ellipsoïde des indices dont les axes principaux coïncident avec les directions principales des contraintes. Ainsi lorsque le rayon lumineux atteint le verre, il se divise immédiatement en deux vibrations dont les orientations suivent les deux directions principales secondaires, c'est-à-dire les deux directions relatives au plan, dans le matériau, perpendiculaire à l'axe de propagation de la lumière. La troisième direction ne contribue pas à la biréfringence puisque la vitesse de propagation d'un rayon de lumière n'est pas régie par l'alignement de matière le long de ce rayon, mais par la nature du milieu normalement au rayon.

Le retard relatif de phase $\Delta$ qui se crée entre les deux vibrations suit les équations de Neumann [10] :

$$
\left\{\begin{array}{l}
\frac{\mathrm{d} \Delta}{\mathrm{d} x_{3}}=\mathrm{C}\left(\sigma_{1}-\sigma_{2}\right)+2 \frac{\mathrm{d} \varphi}{\mathrm{d} x_{3}} \sin \Delta \cot \kappa \\
\frac{\mathrm{d} \kappa}{\mathrm{d} x_{3}}=\frac{-\mathrm{d} \varphi}{\mathrm{d} x_{3}} \cos \Delta
\end{array}\right.
$$


$\kappa$ est tel que $\tan \kappa=\frac{\mathrm{E}_{2}}{\mathrm{E}_{1}}$ où $\mathrm{E}_{1}$ et $\mathrm{E}_{2}$ sont les amplitudes de la vibration lumineuse le long des directions principales secondaires $\left(\mathrm{E}_{1}>\mathrm{E}_{2}\right)$.

$\sigma_{1}$ et $\sigma_{2}$ sont les contraintes principales secondaires (dans le plan de la plaque, c'est-à-dire sans tenir compte de la troisième direction qui ne contribue pas à la biréfringence), $\varphi$ est l'angle des directions principales secondaires avec l'axe des polariseurs et C la constante photoélastique du verre.

Dans le cas de la polariscopie circulaire, après avoir traversé le verre, le rayon lumineux se propage au travers d'une seconde lame quart d'onde, dont les axes rapide et lent sont opposés à ceux de la première lame. Le second polariseur, appelé analyseur, ramène les deux vibrations dans le même plan et les fait donc s'interférer. L'intensité I de la lumière transmise s'écrit :

$$
\begin{cases}\mathrm{I}=\mathrm{I}_{0} \sin ^{2} 2 \varphi \sin ^{2}(\Delta / 2) & \text { si polariscopie plane } \\ \mathrm{I}=\mathrm{I}_{0} \sin ^{2}(\Delta / 2) & \text { si polariscopie circulaire }\end{cases}
$$

Pour une polariscopie plane, cette intensité s'annule si $\varphi \equiv 0(\pi / 2)$ ou $\Delta \equiv 0(2 \pi)$.

Dans le premier cas, les franges noires s'appellent les isoclines; elles correspondent aux lieux des points où les directions principales secondaires sont égales aux directions des axes des polariseurs.

Dans le second cas, les franges noires s'appellent les isochromes. En lumière blanche, chaque retard de phase correspond à une couleur bien précise. Les isochromes sont d'autant plus nombreuses que la différence entre les contraintes principales secondaires est grande.

Pour une polarisation circulaire, seules les isochromes apparaissent, empêchant ainsi la superposition des deux types de franges noires, ce qui peut provoquer des problèmes dans les interprétations notamment en lumière monochromatique.

\subsection{Simulation des images photoélastiques}

\subsubsection{Discussion}

Le retard de phase $\Delta$ dépend des contraintes principales secondaires, et donc de l'état de contraintes, par l'intermédiaire des équations de Neumann (1).

Les contraintes principales secondaires $\sigma_{1}$ et $\sigma_{2}$ sont connues grâce à la simulation par Éléments Finis présentée précédemment. Ainsi la résolution de ce système complet permet de déterminer le retard de phase $\Delta$ et donc l'intensité de la lumière. Les images photoélastiques peuvent être simulées par ce principe. Toutefois, la résolution des équations de Neumann est complexe, c'est un système d'équations différentielles non linéaires à coefficients non constants.

Le système se simplifie si l'angle $\varphi$ ne dépend pas de $x_{3}$, la coordonnée relative à la direction de propagation du rayon lumineux, c'est-à-dire si les directions principales secondaires restent toujours les mêmes le long du trajet optique. Dans ce cas, le système (1) devient :

$$
\frac{\mathrm{d} \Delta}{\mathrm{d} x_{3}}=\mathrm{C}\left(\sigma_{1}-\sigma_{2}\right)
$$

On se ramène alors au cas de la photoélasticité $2 \mathrm{D}$, obtenu notamment pour une plaque de verre non trouée, placée dans un polariscope dont l'axe est normal au plan de la plaque, et qui est chargée dans son plan.

Or, dans les zones de connexion des structures en verre, la présence d'un trou à géométrie complexe ne permet pas une telle hypothèse. Il n'est plus possible de se ramener à une structure $2 \mathrm{D}$ en raison des chanfreins dans ces zones. C'est à $45^{\circ}$ par rapport à la direction de chargement que le cisaillement $\sigma_{12}$ est le plus important, ce cisaillement n'étant pas constant le long du trajet optique. Ainsi, les directions principales seront amenées à tourner, et le terme $\frac{\mathrm{d} \varphi}{\mathrm{d} x_{3}}$ ne pourra pas être négligé. Les équations de Neumann (1) doivent être considérées dans leur ensemble pour notre cas particulier.

Il est possible de montrer que ce système est équivalent au système matriciel suivant, obtenu à partir des équations électromagnétiques de Maxwell [10] :

$$
\begin{aligned}
& \frac{\mathrm{d}[\mathrm{E}]}{\mathrm{d} x_{3}}= \\
& \quad-\frac{\mathrm{i} \pi \mathrm{C}}{\lambda}\left[\begin{array}{ll}
\sigma_{11}\left(x_{3}\right)-\sigma_{22}\left(x_{3}\right) & 2 \sigma_{12}\left(x_{3}\right) \\
2 \sigma_{12}\left(x_{3}\right) & -\left(\sigma_{11}\left(x_{3}\right)-\sigma_{22}\left(x_{3}\right)\right)
\end{array}\right][\mathrm{E}]
\end{aligned}
$$

que l'on notera :

$$
\frac{\mathrm{d}[\mathrm{E}]}{\mathrm{d} x_{3}}=\mathrm{M}\left(x_{3}\right) \cdot[\mathrm{E}]
$$

[E] désigne le vecteur champ électrique, $\lambda$ est la longueur d'onde de la lumière, $\sigma_{\mathrm{ij}}$ sont les composantes du tenseur des contraintes dans le plan perpendiculaire à la direction de propagation de la lumière.

\subsubsection{Principe}

L'intégration de cette équation différentielle peut être réalisée à l'aide d'un schéma de Cranck-Nicholson [6] :

$$
\frac{\mathrm{E}\left(x_{3}+\Delta x_{3}\right)-\mathrm{E}\left(x_{3}\right)}{\Delta x_{3}}=\mathrm{M}\left(x_{3}\right)\left(\frac{\mathrm{E}\left(x_{3}+\Delta x_{3}\right)+\mathrm{E}\left(x_{3}\right)}{2}\right)
$$

Ce qui donne l'expression du vecteur E à l'altitude $x_{3}+$ $\Delta x_{3}$ à partir de sa valeur à l'altitude $x_{3}$ ( $\mathrm{I}_{\mathrm{d}}$ est la matrice identité) :

$$
\begin{aligned}
& \mathrm{E}\left(x_{3}+\Delta x_{3}\right)= \\
& \quad\left[\mathrm{I}_{\mathrm{d}}-\frac{\Delta x_{3}}{2} \mathrm{M}\left(x_{3}\right)\right]^{-1} \cdot\left[\mathrm{I}_{\mathrm{d}}+\frac{\Delta x_{3}}{2} \mathrm{M}\left(x_{3}\right)\right] \cdot \mathrm{E}\left(x_{3}\right)
\end{aligned}
$$

Ainsi, nous ne faisons plus l'hypothèse d'invariance des directions principales secondaires dans l'épaisseur de 
la plaque (invariance du cisaillement dans le plan de la plaque suivant la direction 3). Cette hypothèse vient de la diagonalisation de la matrice $\mathrm{M}$, qui fait apparaître la matrice de changement de repère où intervient l'angle $\varphi$ des directions principales secondaires, considéré indépendant de $x_{3}$.

Le choix de la méthode de mesure, polariscopie circulaire ou polariscopie rectiligne, conditionne dans ces calculs la valeur initiale du vecteur $\mathrm{E}_{0}$, et de l'analyseur final. Comme seul compte le rapport de l'intensité lumineuse transmise sur l'intensité incidente, la norme du vecteur est arbitrairement fixée $\mathrm{E}_{0}$ à 1 au début. La valeur de l'intensité lumineuse après passage dans l'analyseur vaut :

$$
\mathrm{I}_{\text {transmise }}=(\langle\mathrm{E}, \mathrm{A}\rangle)^{2}
$$

A est un vecteur dont la direction est donnée par l'axe de l'analyseur.

Ainsi, selon les montages, nous obtenons :

- En polarisation circulaire

$$
\mathrm{E}_{0}=\left(\begin{array}{r}
\mathrm{i} / \sqrt{2} \\
-1 / \sqrt{2}
\end{array}\right) \quad \mathrm{A}=\left(\begin{array}{c}
-1 / \sqrt{2} \\
-\mathrm{i} / \sqrt{2}
\end{array}\right)
$$

- En polarisation rectiligne :

- si le polariseur fait un angle nul avec l'horizontale :

$$
\mathrm{E}_{0}=\left(\begin{array}{r}
0 \\
-1
\end{array}\right) \quad \mathrm{A}=\left(\begin{array}{r}
-1 \\
0
\end{array}\right)
$$

- si le polariseur fait un angle de $+45^{\circ}$ avec l'horizontale :

$$
\mathrm{E}_{0}=\left(\begin{array}{r}
1 / \sqrt{2} \\
-1 / \sqrt{2}
\end{array}\right) \quad \mathrm{A}=\left(\begin{array}{l}
-1 / \sqrt{2} \\
-1 / \sqrt{2}
\end{array}\right)
$$

L'intensité I de la lumière est égale au carré de la norme du vecteur champ électrostatique et est reliée au retard de phase $\Delta$ par l'expression donnée précédemment.

Un programme, écrit en FORTRAN, a été réalisé [6]. Ce programme lit le fichier de données généré par ABAQUS (état de contraintes en chaque point de Gauss) et permet de calculer :

- le retard de phase et comparer les isovaleurs prédites aux images obtenues expérimentalement en lumière blanche (dans le cas de la lumière polarisée rectilignement, l'angle $\varphi$ s'obtient en cherchant les vecteurs propres de la matrice $2 \times 2$ des contraintes relatives au plan de la plaque),

- ou l'intensité de la lumière et rechercher les lieux d'extinction (c'est-à-dire où l'intensité est nulle) pour les comparer aux isochromes obtenues en lumière monochromatique (une seule longueur d'onde).

\subsubsection{Validation}

Afin de juger la validité de ce programme d'analyse d'images photoélastiques, les résultats de prédiction des images sont comparés à des résultats expérimentaux.

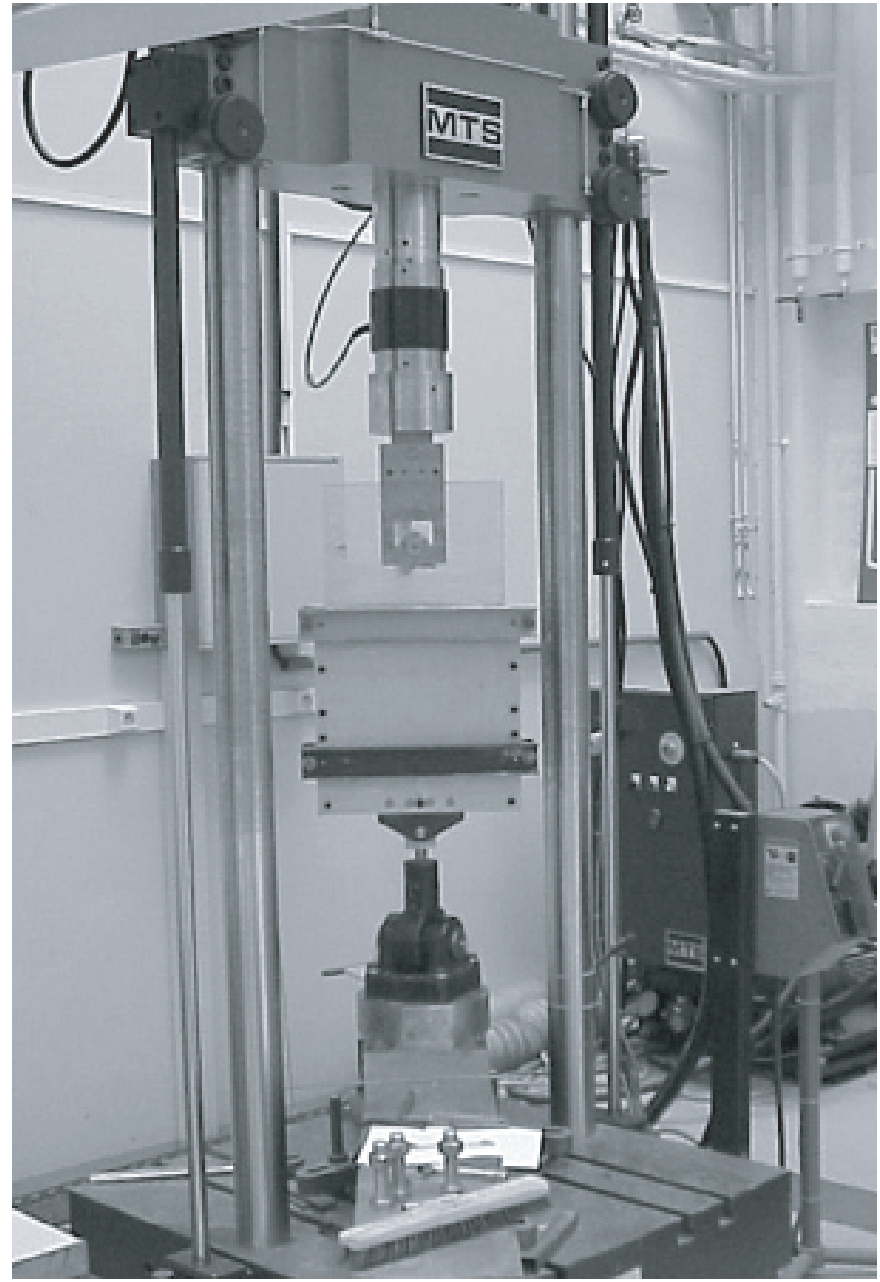

Fig. 7. Montage des essais réalisés pour la détermination de la résistance ultime de plaques de verre trouées.

La campagne expérimentale réalisée vise à déterminer les charges ultimes dans des zones d'assemblages de structures en verre. C'est en effet à cet endroit que les états de contraintes sont les plus complexes, la validation de l'approche sera donc pertinente. La figure 7 est une photographie d'un des essais réalisés. La plaque de verre utilisée fait $350 \mathrm{~mm}$ de large, $600 \mathrm{~mm}$ de haut. L'axe du trou est à $125 \mathrm{~mm}$ du haut de la plaque. L'épaisseur est de $19 \mathrm{~mm}$ (épaisseur généralement maximale produite par les industriels, nécessaire dans le domaine du verre structurel).

Le verre est collé à deux flasques métalliques, reliées à une rotule fixée au bâti de la machine d'essais (MTS 50 tonnes). Le connecteur métallique est attaché à deux autres plaques fixées à la traverse horizontale de la machine d'essais. Il est ainsi assujetti à se déplacer verticalement, vers le haut à une vitesse contrôlée. Loin du trou l'état de contraintes dans le verre est un état de traction. Le chargement est bien dans le plan de la plaque. La présence de la rotule assure la non-existence d'effort de torsion et de flexion.

Cinq géométries de trous ont été testées (Fig. 8 et Tab. 1). 


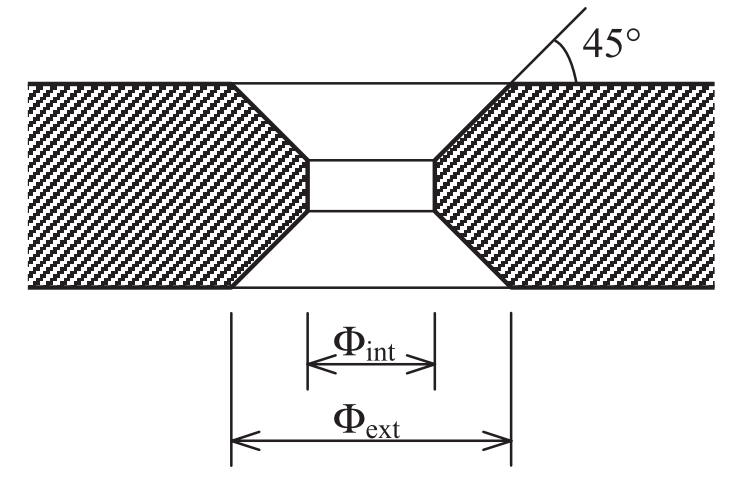

Fig. 8. Géométrie des trous.
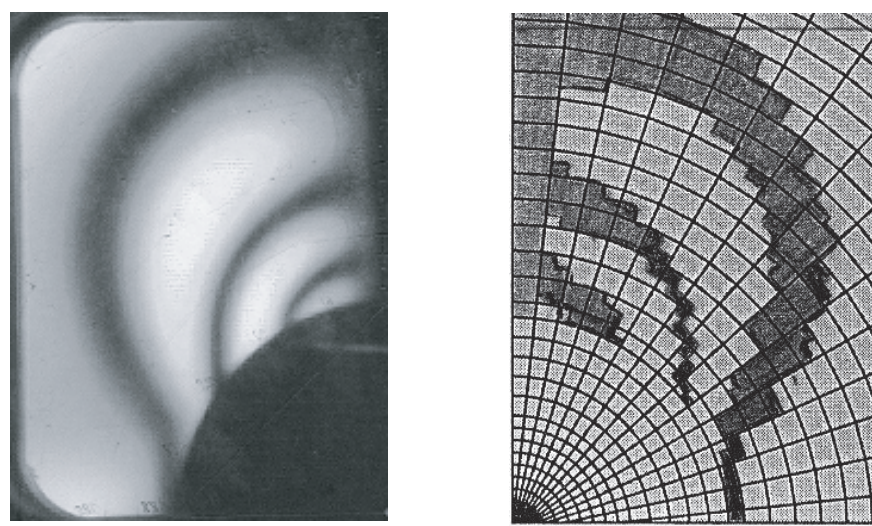

Fig. 9. Images photoélastiques observée et calculée pour une plaque de type b1 et pour un chargement de $20 \mathrm{kN}$.

Tableau 1. Dimensions des 5 géométries de trous testés.

\begin{tabular}{ccc}
\hline Désignation & $\Phi_{\text {int }}(\mathrm{mm})$ & $\Phi_{\text {ext }}(\mathrm{mm})$ \\
\hline a1 & 38 & 40 \\
a2 & 54 & 56 \\
b1 & 24 & 40 \\
b2 & 40 & 56 \\
c1 & 30 & 40 \\
\hline
\end{tabular}

Un orifice est aménagé dans la plaque permettant de charger le connecteur métallique. C'est dans cette zone, située juste au-dessus et sur les côtés du trou que les mesures photoélastiques avec un polariscope ont été effectuées. Les figures 9 et 10 montrent les comparaisons entre les images photoélastiques visualisées au cours d'un essai pour un chargement donné et une géométrie donnée, avec les images photoélastiques simulées à partir de l'état de contraintes obtenu par le calcul par Éléments Finis pour le même chargement.

Les résultats de la simulation sont donc en bon accord avec les résultats expérimentaux. Cela valide à la fois la simulation numérique du chargement du connecteur métallique et le programme d'analyse d'images photoélastiques développés.
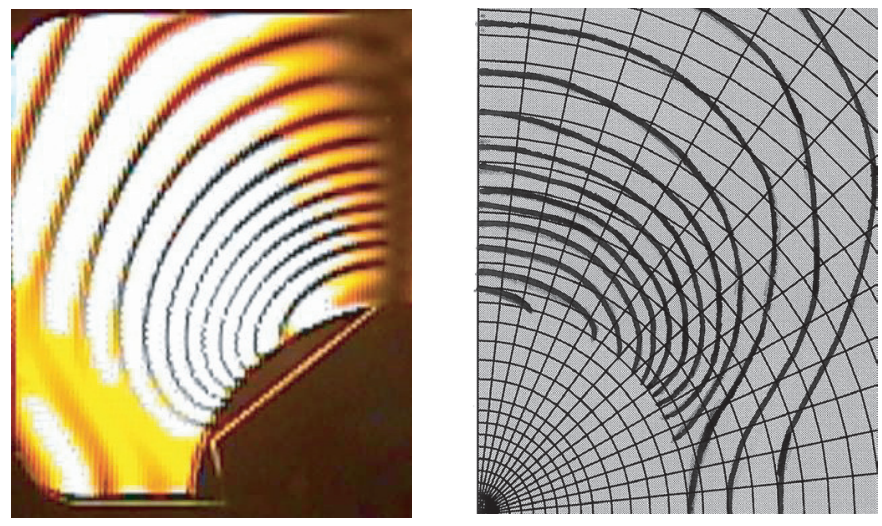

Fig. 10. Images photoélastiques observée et simulée pour un trou de type $\mathrm{c} 1$ et pour un chargement de $94 \mathrm{kN}$.

\subsubsection{Utilisation pour le contrôle in situ}

Il semble maintenant possible de passer d'un état de contraintes à une image photoélastique, et réciproquement. Ainsi, en analysant des images photoélastiques obtenues sur des structures existantes, l'état de contraintes dans le matériau peut être prédit. Il est également possible de vérifier une possible décompression de la surface par analyse inverse ou quantifier l'écart de contraintes avant cette décompression, et être en mesure de dresser ou non des certificats de garantie.

Les décompressions de surface sont calculées grâce à la simulation par Éléments Finis détaillée précédemment. Par exemple pour des zones d'assemblage comportant un trou de type b2, avec un chargement dans le plan de la plaque de verre et un pré-serrage de 2 daN.m, la surface se décomprime à partir de $80 \mathrm{kN}$ [6]. Un cœefficient de sécurité de 3.5, intégrant l'incertitude sur les charges et le caractère fragile du matériau, est couramment utilisé pour de telles applications. Avec un tel coefficient de sécurité, la structure ne devra pas supporter, en service, au niveau du trou, une charge supérieure à $80 / 3.5=22.9 \mathrm{kN}$. Si la méthode actuelle de dimensionnement est utilisée, un cœefficient partiel de sécurité de 2 caractérisant le comportement à long terme du matériau devra également être pris en compte et le chargement appliqué sur le trou ne devra pas excéder le septième de la charge à rupture $(112 \mathrm{kN}$ d'après [6]), soit $112 / 7=16 \mathrm{kN}$.

La figure 11 présente alors les images photoélastiques simulées correspondant :

- à la charge de décompression de la surface $(80 \mathrm{kN})$;

- à la charge de décompression de la surface divisée par un cœfficient de sécurité de $3.5(23 \mathrm{kN})$; ceci correspondrait à un assouplissement de la méthode de dimensionnement en vigueur actuellement, que nous préconisons dans cet article;

- à la charge à rupture divisée par un cœefficient de 7 $(16 \mathrm{kN})$; ceci correspond à la méthode de dimensionnement utilisée actuellement.

Suivant la méthode de dimensionnement préconisée, ces images donneront l'état limite admissible. 


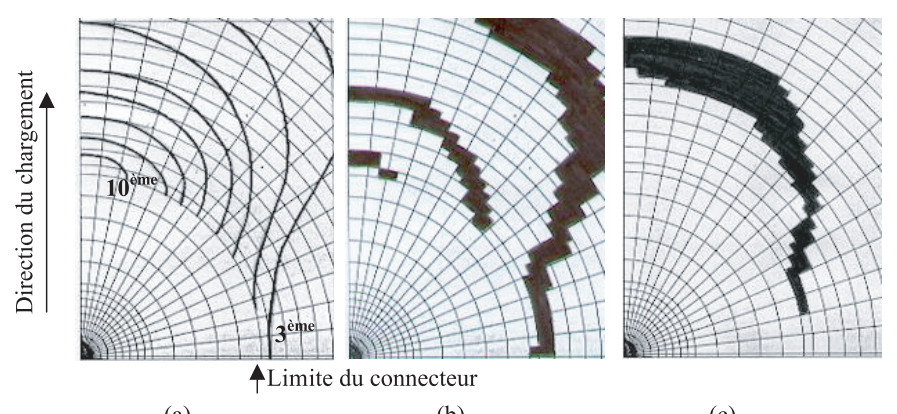

(a) (c)

Fig. 11. Différentes images photoélastiques de la plaque de verre au dessus du connecteur, obtenues par simulation pour une force de $80 \mathrm{kN}$ (a), $23 \mathrm{kN}$ (b), $16 \mathrm{kN}$ (c).

\section{Conclusion}

Ce travail s'inscrit dans le contexte du « verre structurel », c'est-à-dire l'utilisation du verre pour les structures du génie civil. Le manque de connaissances réellement approfondies du comportement mécanique de ce matériau à long terme conduit à le défavoriser pour de telles applications. Des essais en vraie grandeur et des cœefficients de sécurité élevés sont exigés par les bureaux de contrôle pour la validation de la construction.

Afin d'assouplir ces principes actuellement en vigueur, une nouvelle méthode de dimensionnement est proposée. Elle consiste à dimensionner l'ouvrage à l'état limite de service en prenant comme charge limite la charge de décompression de la surface des éléments en verre. La détermination de celle-ci nécessite d'avoir recours à la simulation par Éléments Finis, pour calculer d'une part l'état de contraintes résiduelles après trempe, et d'autre part (ce qui est complexe essentiellement pour la zone de connexion) les états de contraintes engendrées par des chargements extérieurs s'appliquant sur la structure en verre.

L'utilisation de la photoélasticité est proposée pour le contrôle in situ des structures existantes. La simulation des images photoélastiques, qui permet de relier état de contraintes et franges isochromatiques, est présentée

et développée. L'originalité du travail présenté est la prise en compte du caractère tridimensionnel de l'état de contraintes dans les zones de connexion. Le programme d'analyse d'images écrit dans cette étude permet ainsi l'intégration d'une possible rotation des directions principales secondaires dans l'épaisseur de la plaque c'est-à-dire le long du trajet optique de la lumière. Cette méthode permettra de vérifier in situ l'état de la structure en verre : notamment chercher à savoir si la surface du verre est décomprimée ou non, et donc d'être en mesure de dresser des certificats de garantie.

\section{Références}

[1] T.A. Michalske, S.W. Freiman, A molecular mechanism for stress corosion in vitreous silica, J. American Ceramic Society 66(4) (1983) 284-288

[2] R. Gy, Creep and recovery of a thermally tempered glass plate at room temperature, in J. Vitkala (ed.), Proceedings of Glass Processing Days, Tamglass Ltd, Helsinki, Finland, 1999

[3] P. Rice, H. Dutton, Le Verre Structurel, $2^{\mathrm{e}}$ édition, Publications du Moniteur, Paris, 1995

[4] O.S. Narayanaswamy, A model of structural relaxation in Glass, J. American Ceramic Society 54(10) (1971) 491-498

[5] H. Carré, L. Daudeville, Load bearing capacity of tempered structural glass, ASCE J. Eng. Mech. 125(8) (1999) 914-921

[6] F. Bernard, Sur le dimensionnement des structures en verre trempé : Étude des zones de connexion, Thèse, École normale supérieure de Cachan, 2001

[7] F. Bernard, R. Gy, Daudeville L., Finite element computation of residual stresses near holes in tempered glass plates, Glass Technology 43C (2002) 290-295

[8] S. Lin, D.A. Hills, Nowell D. Stresses in a flat plate due to a loose pin pressing against a crack hole, J. Strain Analysis 32(2) (1997) 145-156

[9] ABAQUS/ Standard : User's manual, version 5.8, Hibbitt, Karlson \& Sorensen Inc. (1998)

[10] H. Aben, C. Guillemet, Photoelasticity of glass, SpringerVerlag, Berlin, 1993 